(C)2008 IEEE. Personal use of this material is permitted. However, permission to reprint/republish this material for advertising or promotional purposes or for creating new collective works for resale or redistribution to servers or lists, or to reuse any copyrighted component of this work in other works must be obtained from the IEEE. 


\title{
Derivation of a New Mathematical Framework for Transmission System Augmentation using von Stackelberg Game
}

\author{
M. R. Hesamzadeh, N. Hosseinzadeh, and P. J. Wolfs, CQ University, Australia
}

\begin{abstract}
The market-based augmentation of the high voltage transmission systems as a legacy of the previous regulated regimes has been a challenging issue for the central transmission entities. The economic assessment framework for transmission upgrades or expansion projects, considering the interaction of the central transmission entity (CTE) with the electricity market management (MMC) company both as independent players needs to be addressed appropriately at least in the National Electricity Market, Australia.

To assist in bridging this gap, this paper introduces a novel metric, namely, the L-Shape Area, for the economic assessment of the transmission expansion options. The proposed methodology employs a von Stackelberg game for the interaction modelling of the central transmission entity and the market management company. The upper subproblem minimises the objective of the CTE as the leader player and the lower subproblem solves the security-constrained economic dispatch as the follower subproblem.

The bi-level programming problem has been solved by applying the Kuhn-Tucker optimality conditions to the follower subproblem and the use of a gradient search method to solve the resultant single level non-linear programming problem.

A modified IEEE 14-bus test system has been used to show the effectiveness of the proposed formulation.
\end{abstract}

Index Terms - High Voltage Transmission System, MarketBased Augmentation, von Stackelberg Game

\section{INTRODUCTION}

The National Electricity Market, Australia, (NEM) is a gross pool with Queensland, New South Wales, Australian Capital Territory, Victoria, South Australia, and Tasmania as participating jurisdictions. Generators sell their electricity to the pool and customers buy their required electric energy from the pool. The main customers are retailers which buy electricity to resell it to the household and business customers. While it is possible for an end-use customer to buy directly from the pool, few choose this option [1]. The National Electricity Management Company is the market operator [2].

The NEM in eastern and southern Australia has a combination of state-based transmission networks and crossborder interconnectors that connect the states together [3]. The Queensland transmission system with the total line length of around $11902 \mathrm{~km}$ and the maximum demand of $8232 \mathrm{MW}$ is one of the longest transmission systems in the NEM. Powerlink is a regulated monopoly business responsible for the augmentation of the high voltage transmission system in
Queensland to providing all NEM participants with open and non-discriminatory access [2].

Powerlink, as the Central Transmission Entity (CTE), is in charge of transmission planning and operation within the state of Queensland. This central transmission entity bears any transmission investment cost. Unlike the reliability-based planning approach, the market-based augmentation approach has not been accommodated properly in the Queensland transmission planning methodology.

In [4], the author has done a peer review on the available methodologies for the market-based augmentation of high voltage transmission systems. Development of a comprehensive metric for measuring the market value of the transmission expansion options is the very critical issue. This issue must be addressed as the first step in market-based augmentation of the high voltage transmission systems. Accordingly, in [5], the author has done an analytical review on the most comprehensive metrics used in the economic assessment of transmission expansion projects in competitive electricity markets. An exact definition along with its mathematical formulation has been provided for each introduced metric. In [5], around 100 papers have been reviewed and 40 of them have been discussed. Aggregated surplus, congestion revenue, market players' surplus, redispatch power, and redispatch cost have been used as the metrics for the electricity market efficiency measuring. Flatness of price profile, Lerner index, Quantity weighted Lerner index, Residual Supply index, and HerfindahlHirshman index have been employed for the measuring of competitiveness in electricity market. As it is clear, these indices can evaluate electricity market only based on either efficiency or competitiveness. The lack of a comprehensive metric for accommodating the efficiency and competitiveness of electricity market is clear from above survey.

On the other hand the interaction of central transmission entity and market management company in the market-based augmentation of transmission system has not received enough attention.

Covering the aforementioned shortcomings, this paper proposes a novel metric, namely, the L-Shape Area criterion as a measure of the performance of the electricity market. The CTE and MMC interaction has been modelled as the leaderfollower von Stackelberg game. The leader is the CTE with the objective function of the investment cost of transmission 
upgrade or expansion project and the L-Shape Area criterion. The security-constrained economic dispatch is solved as the MMC's central dispatch process. This paper is organised in five sections and two appendices. The mathematical formulation of the problem is detailed in section II. Section III deals with the solution of the problem using the von Stackelberg game. This section also includes the Kuhn-Tucker optimality conditions and gradient search method in solving the resultant bi-level programming problem.

\section{THE MATHEMATICAL FORMULATION OF THE PROBLEM}

\section{A. L-Shape Area Metric}

The introduction of competitive markets aims to reduce prices, improve the quality of services, and on a long-term basis make the market more efficient [6]. Providing an environment for perfect competition between generators on one side of market and between retailers on the other side is the necessary condition in achieving the aforementioned targets.

The conditions required for perfect competition are, [7]:

(1) a large number of generators producing the same product;

(2) each generator attempts to maximise its payoff;

(3) each generator is a price taker;

(4) generators have exact knowledge of all parameters of significance to their decisions;

(5) transmission is costless.

The first condition aims to prevent the formation of market concentration in terms of Herfindahl-Hirshman Index and pivotal generators in terms of Residual Supply Index [8]. Having rational generators is the immediate consequence of second condition, [9]. Given rational generators, when a generator is a price taker, mathematically it can be shown that maximising its profit requires bidding its marginal cost, [10]. Condition four says that the generators have perfect information. Finally, the last condition addresses bottlenecks in the high voltage transmission systems. Bottlenecks are one of the major issues in market separation. Arguably, none of these conditions ever exists in a real electricity market. Accordingly, the real electricity market has deviation from the perfect competitive electricity market. Measuring this deviation has been addressed in a few publications with some degree of success.

This paper proposes a novel metric for measuring the performance of the electricity market. The L-Shape area criterion, $A_{L-S h a p e}$, can accommodate the efficiency and competitiveness measuring of the electricity market in one comprehensive metric. The L-Shape area metric is designed based on measuring the deviation of the real electricity market from an ideal one. This paper defined an ideal electricity market as one which satisfies all five previous explained conditions.
In terms of mathematical derivation throughout this paper the bold letters enclosed by [] are matrices, bols letters are vectors and unbold letters are scalar quantities.

Definition of L-Shape Area Metric ( $\left.A_{L-S h a p e}\right)$ : Let $\boldsymbol{\lambda}_{\boldsymbol{c}}$ and $\boldsymbol{\lambda}$ be the nonempty vectors in $\mathcal{R}^{n}$ representing nodal prices $(\$ / \mathrm{MWh})$ and let $D_{\max } \neq 0$ and $D \neq 0$ be the aggregated served demand of retailers (MWh) in ideal and real electricity market respectively. If $\Delta \lambda$ is the Euclidean norm of the vector $\lambda_{c}-\lambda$ as in (1),

$$
\begin{aligned}
& \Delta \lambda=\left\|\lambda-\lambda_{c}\right\|_{2} \\
& \text { then } A_{L-S h a p e} \geq 0(\$ / \mathrm{h}) \text { is; } \\
& A_{L-\text { Shape }}=D_{\max }\left(\lambda_{c}+\Delta \lambda\right)-D \lambda_{c}(2)
\end{aligned}
$$

Note that all elements of vector $\lambda_{c}$ are the same and equal to $\lambda_{c}$.

The $A_{L-\text { Shape }}$ is depicted in fig. 1.

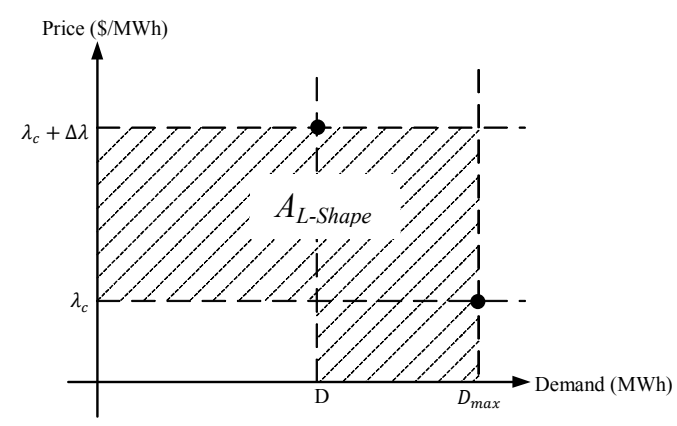

Fig. 1, L-Shape Area Metric

Based on the $A_{L \text {-Shape }}$ criterion, the following theorem can be developed.

Theorem: The necessary and sufficient condition for perfect competition in an electricity market is that:

$$
A_{\text {L-Shape }}=0
$$

Proof: see appendix I.

Subsection B deals with mathematical modelling of the central transmission entity as the leader player.

\section{B. Central Transmission Entity (CTE)}

Suppose a $C T E$ has $m$ upgrade options and $n$ expansion options for the market-based augmentation of the high voltage transmission system in its given territory. For the $m$ upgrade options, $\boldsymbol{f}_{l}^{\prime \boldsymbol{u}}, l=1, \ldots, m$ and for the $n$ expansion options, $\boldsymbol{f}_{l}^{\prime \boldsymbol{e}}$ $, l=1, \ldots, n$ are the vectors of maximum thermal capacity which can be built on the transmission corridors of upgrade and expansion, respectively. Similarly, $\boldsymbol{t} \boldsymbol{c}_{\boldsymbol{l}}^{\prime \boldsymbol{u}}, l=1, \ldots, m$ and $\boldsymbol{t} c_{l}^{\prime e}, l=1, \ldots, n$ are the vectors of the investment cost for the transmission upgrade and expansion projects, respectively. Figure 2 shows this situation. 


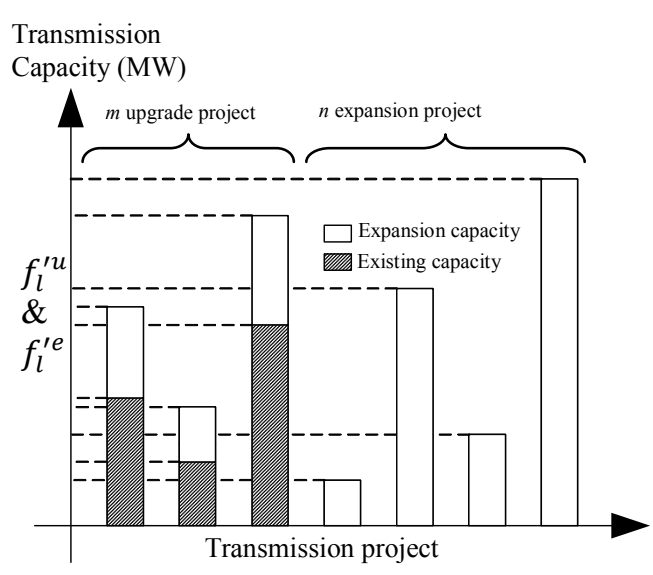

Fig.2, CTE's transmission projects

Since the CTE pays the investment cost of upgrade or expansion, it is desirable to upgrade and/or expand the transmission system with the minimum cost. Mathematically, the CTE's cost function can be formulated as (4).

$$
\begin{gathered}
\operatorname{Min} \Pi=\llbracket \alpha A_{L-\text { Shape }}+\beta\left(\sum_{l=1}^{m} f_{l}^{\prime u} t c_{l}^{\prime u}+\sum_{l=m+1}^{m+n} f_{l}^{\prime e} t c_{l}^{\prime e}\right) \rrbracket \\
\text { s.t. } \\
0 \leq f_{l}^{\prime u} \leq \bar{f}_{l}^{\prime u} l=1, \ldots, m \\
0 \leq f_{l}^{\prime e} \leq \bar{f}_{l}^{\prime e} l=m+1, \ldots, m+n
\end{gathered}
$$

$\bar{f}_{l}^{\prime u}$ and $\bar{f}_{l}^{\prime e}$ are the maximum values for the $f_{l}^{\prime u}$ and $f_{l}^{\prime e}$ as the CTE's design parameters. $\alpha$ and $\beta$ where $\alpha+\beta=1$ are the weighting factors which are set by the design engineer based on his judgement on the value of transmission investment compared with the value of electricity market performance.

Market clearing process as the main responsibility of MMC is detailed in subsection $\mathrm{C}$.

\section{Market Management Company (MMC)}

Considering the National Electricity Market in Australia, suppose that the electricity market is a double-sided gross pool. MMC collects the bids and uses a security-constrained economic dispatch process for clearing market. With $N_{G}$ registered generators and $N_{R}$ registered retailers in the market, vectors $\boldsymbol{C}^{\prime}$ and $\boldsymbol{V O} \boldsymbol{L} \boldsymbol{L}$ are the apparent marginal cost of $N_{G}$ generators and the value of lost load for the $N_{R}$ retailers. Accordingly, the mathematical formulation of the securityconstrained economic dispatch has been given in (5).

$$
\begin{gathered}
\text { Min } \boldsymbol{C}^{\prime} . \boldsymbol{g}-\boldsymbol{V O L L . d} \\
\text { s.t. } \\
{\left[\mathcal{B}_{x}^{\prime}\right] \boldsymbol{\theta}=\boldsymbol{g}-\boldsymbol{d}} \\
-\left(\boldsymbol{f}_{l}^{0}+\boldsymbol{f}_{l}^{\prime \boldsymbol{u}}+\boldsymbol{f}_{l}^{\prime \boldsymbol{e}}\right) \leq\left[\mathcal{H}_{l}^{\prime}\right] \boldsymbol{\theta} \leq \boldsymbol{f}_{l}^{0}+\boldsymbol{f}_{l}^{\prime \boldsymbol{u}}+\boldsymbol{f}_{l}^{\prime \boldsymbol{e}} \\
\underline{\boldsymbol{g}} \leq \boldsymbol{g} \leq \overline{\boldsymbol{g}} \\
\underline{\boldsymbol{d}} \leq \boldsymbol{d} \leq \overline{\boldsymbol{d}}(5)
\end{gathered}
$$

In (5), $\left[\mathcal{B}_{x}^{\prime}\right]$ and $\left[\mathcal{H}_{l}^{\prime}\right]$ are $N_{b} \times\left(N_{b}-1\right)$ and $N_{l} \times\left(N_{b}-1\right)$ matrix where the column related to the slack bus is omitted, $N_{b}$ and $N_{l}$ are the total number of buses and total number of lines in the system. $\boldsymbol{\theta}$ is the vector of bus angles and $\boldsymbol{g}$ and $\boldsymbol{d}$ are the generation level of committed generators and the served demand of retailers. $\boldsymbol{\theta}, \boldsymbol{g}$, and $\boldsymbol{d}$ act as the decision variable of (5). These variables are bounded by their minimum and maximum values. Existing capacity of the transmission system has been modelled through vector $\boldsymbol{f}_{\boldsymbol{l}}^{\mathbf{0}}$.

The interaction of CTE and MMC for the market-based augmentation of the transmission system has been modelled through a bilevel optimisation problem. The leader makes its decision and the follower takes the leader's decision and optimises its situation. Section III deals with the mathematical formulation of the market-based augmentation based on a von Stackelberg game. Solution of the problem using a modified gradient search method is detailed afterward.

\section{VON STACKELBERG GAMING WITH COMPLETE INFORMATION}

The static version of the non-cooperative, two-person game introduced by von Stackelberg, [11], is used for the marketbased augmentation of the high voltage transmission system. Perfect information is assumed so that both players, CTE and MMC, know the objective and feasible choices available to the other. The fact that the game is said to be static implies that each player has only one move. CTE moves first and minimises its objective function taking into account all possible responses from MMC, the MMC observes the CTE's decision and clears the market by solving (5).

The complete problem formulation has been given in (6).

$$
\begin{gathered}
\text { Min } \Pi=\llbracket \alpha A_{L-\text { Shape }}+\beta\left(\sum_{l=1}^{m} f_{l}^{\prime u} t c_{l}^{\prime u}+\sum_{l=m+1}^{m+n} f_{l}^{\prime e} t c_{l}^{\prime e}\right) \rrbracket \\
\text { s.t. } \begin{array}{c}
0 \leq f_{l}^{\prime u} \leq \bar{f}_{l}^{\prime u} l=1, \ldots, m \\
0 \leq f_{l}^{\prime e} \leq \bar{f}_{l}^{\prime e} l=m+1, \ldots, m+n
\end{array}
\end{gathered}
$$

$$
\begin{aligned}
& \text { Min } \boldsymbol{C}^{\prime} . \boldsymbol{g}-\boldsymbol{V O} \boldsymbol{O L L . d} \\
& \text { s.t. } \\
& \qquad \begin{aligned}
& {\left[\mathcal{B}_{x}^{\prime}\right] \boldsymbol{\theta}=\boldsymbol{g}-\boldsymbol{d} } \\
&-\left(\boldsymbol{f}_{l}^{0}+\boldsymbol{f}_{l}^{\prime \boldsymbol{u}}+\boldsymbol{f}_{l}^{\prime e}\right) \leq\left[\mathcal{H}_{l}^{\prime}\right] \boldsymbol{\theta} \leq \boldsymbol{f}_{l}^{0}+\boldsymbol{f}_{l}^{\prime u}+\boldsymbol{f}_{l}^{\prime e} \\
& \underline{\boldsymbol{g}} \leq \boldsymbol{g} \leq \overline{\boldsymbol{g}} \\
& \underline{\boldsymbol{d}} \leq \boldsymbol{d} \leq \overline{\boldsymbol{d}}(6)
\end{aligned}
\end{aligned}
$$

Programming problem (6) can be categorised as the nonlinear bilevel programming problem. Generally, non-linear bilevel programming programs are intrinsically hard [12]. Even the simplest form of the linear-linear bilevel programming problems has been proved to be $\mathcal{N} \mathcal{P}$-hard by [13] and strong $\mathcal{N} \mathcal{P}$-hard by [14]. 
Branch and bound algorithm [15], steepest descent direction [16], ,sensitivity and gradient method [17], and heuristic methods have been proposed for solving bilevel programming problem. This paper employs the gradient search method for solving the bilevel programming problem. (7).

The MMC's central dispatch process can be generalized in

$$
\begin{gathered}
\text { Min C. } \boldsymbol{x} \\
\text { s.t. } \\
{[\boldsymbol{B}] \boldsymbol{x}+\boldsymbol{D}_{\mathbf{1}}+\boldsymbol{D}_{\mathbf{2}}+\boldsymbol{D}_{3} \leq \mathbf{0}} \\
{[\boldsymbol{A}] \boldsymbol{x}=\mathbf{0}(7)}
\end{gathered}
$$

The introduced vectors and matrices in (7) in terms of vectors and matrices in (6) are defined in appendix II.

The Kuhn-Tucker optimality conditions [18], [19] for (7) yield to the following sets of equalities.

$[B] x+D_{1}+D_{2}+D_{3}+S=0$

$[A] x=0$

$\nabla(C \cdot x)+\mu \nabla\left([B] x+D_{1}+D_{2}+D_{3}\right)+\lambda \nabla([A] x)=0$

$\mu\left([B] x+D_{1}+D_{2}+D_{3}\right)=0$

$(\boldsymbol{\mu} \geq \mathbf{0}, \boldsymbol{S} \geq \mathbf{0})(8)$

In (8), $\boldsymbol{S}$ is the vector of slack variables.

CTE has two decision vectors, namely, $\boldsymbol{f}_{l}^{\prime \boldsymbol{u}}$ and $\boldsymbol{f}_{l}^{\prime \boldsymbol{e}}$ which are a part of vectors $\boldsymbol{D}_{2}$ and $\boldsymbol{D}_{3}$ respectively. Simplifying (8) and differentiating (8) with respect to $\boldsymbol{f}_{l}^{\prime} \boldsymbol{u}$, we get (9).

$$
\begin{gathered}
{[B] \frac{\partial \boldsymbol{x}}{\partial \boldsymbol{f}_{l}^{\prime u}}+\frac{\partial \boldsymbol{D}_{2}}{\partial \boldsymbol{f}_{l}^{\prime u}}+\frac{\partial \mathcal{S}}{\partial \boldsymbol{f}_{l}^{\prime u}}=\mathbf{0}} \\
{[\boldsymbol{A}] \frac{\partial \boldsymbol{x}}{\partial \boldsymbol{f}_{l}^{\prime u}}=\mathbf{0}} \\
\frac{\partial \boldsymbol{\mu}}{\partial \boldsymbol{f}_{l}^{\prime u}}[B]+\frac{\partial \lambda}{\partial \boldsymbol{f}_{l}^{\prime u}}[\boldsymbol{A}]=\mathbf{0} \\
\frac{\partial \boldsymbol{\mu}}{\partial \boldsymbol{f}_{l}^{\prime u}} \boldsymbol{S}+\boldsymbol{\mu} \frac{\partial \boldsymbol{S}}{\partial \boldsymbol{f}_{l}^{\prime u}}=0
\end{gathered}
$$

Using the transpose properties of $([\boldsymbol{P}]+[\boldsymbol{Q}])^{T}=[\boldsymbol{P}]^{T}+$ $[\boldsymbol{Q}]^{T}$ and $([\boldsymbol{P}][\boldsymbol{Q}])^{T}=[\boldsymbol{Q}]^{T}[\boldsymbol{P}]^{T}$, (9) can be written in the following matrix form;

$$
\left(\begin{array}{ccc}
\boldsymbol{\mu}[\boldsymbol{B}] & -\boldsymbol{S}^{T} & \mathbf{0} \\
{[\boldsymbol{A}]} & \mathbf{0} & \mathbf{0} \\
\mathbf{0} & \boldsymbol{B}^{T} & \boldsymbol{A}^{T}
\end{array}\right)\left(\begin{array}{c}
\frac{\partial \boldsymbol{x}}{\partial \boldsymbol{f}_{l}^{\prime \boldsymbol{u}}} \\
\frac{\partial \boldsymbol{\mu}^{T}}{\partial \boldsymbol{f}_{l}^{\prime \boldsymbol{u}}} \\
\frac{\partial \lambda^{T}}{\partial \boldsymbol{f}_{l}^{\prime \prime}}
\end{array}\right)=\left(\begin{array}{c}
-\boldsymbol{\mu} \frac{\partial \boldsymbol{D}_{2}}{\partial \boldsymbol{f}_{l}^{\prime \prime}} \\
\mathbf{0} \\
\mathbf{0}
\end{array}\right)
$$

In (10), vector $\left(\frac{\partial x}{\partial f_{l}^{\prime \prime}} \frac{\partial \boldsymbol{\mu}^{T}}{\partial f_{l}^{\prime \prime}} \frac{\partial \lambda^{T}}{\partial f_{l}^{\prime \prime}}\right)$ includes the gradient components of $\Pi$ as the leader objective function. In some cases, the rank of left hand side matrix of (10) is lower than the number of variables in (10). In these cases, singular value decomposition methodology has been employed as one of the best methods for solving least-squares problems [20].
The partial derivative of the $\Pi$ with respect to the $f_{l}^{\prime u}$ can be found as in (11).

$$
\begin{gathered}
\frac{\partial \Pi}{\partial f_{l}^{\prime u}}=\frac{D_{\max }}{N_{b} \Delta \lambda} \sum_{i}\left(\lambda_{i}-\lambda_{c}\right) \frac{\partial \lambda_{i}}{\partial f_{l}^{\prime u}}-\lambda_{c} \sum_{j} \frac{\partial d_{j}}{\partial f_{l}^{\prime u}}+t c_{l}^{\prime u} \\
l=1, \ldots, m(11)
\end{gathered}
$$

Since $\Pi$ is not continuously differentiable at $\lambda=\lambda_{c}$, the Euclidian norm $\sqrt{y}$, where $\mathrm{y}$ is the normal definition of second norm, is used as $\sqrt{y+\epsilon} . \epsilon$ is a very small real number ,in our study 0.0001 , that makes $\Pi$ continuously differentiable at $\lambda=\lambda_{c}$.

Using the gradient search, the algorithm starts with an initial guess for the $f_{l}^{\prime u}, l=1, \ldots, m$ and update the value of $f_{l}^{\prime u}$ based on the equation (12).

$$
f_{l}^{\prime u-n e w}=f_{l}^{\prime u-o l d}-\kappa \frac{\partial \Pi}{\partial f_{l}^{\prime u}}(12)
$$

Where $\kappa$ is the step length of movement to the new solution. If the new value of the $f_{l}^{\prime u}$ is not in the specified boundary, the algorithm sets the new value to the upper or lower bound of the $f_{l}^{\prime u}$ based on the violating of upper or lower bound respectively.

$f_{l}^{\prime e}$ as the second decision variable of CTE can be found by the same process.

The iterative process stops when there is no further change in the CTE's decision variables.

\section{CONCLUSIONS AND FUTURE WORKS}

Employing the static version of a von Stackelberg game, a new strategy has been developed for the market-based augmentation of the high voltage transmission systems. The new strategy models the Central Transmission Entity and Market Management Company as two players with their associated cost functions. In defining the central transmission entity's cost function, a novel metric has been developed for the performance measuring of the electricity market. The socalled $A_{L-\text { Shape }}$ criterion can accommodate the efficiency and competitiveness measurement of the electricity market in one comprehensive metric. Although, $A_{L-\text { Shape }}$ has been developed for the market-based augmentation, it can be used as a monetary index for measuring the electricity market performance.

The ultimate goal of the electricity market restructuring is the bridging the generators and retailers through independent transmission companies to provide competition at all levels of the electricity supply chain. Addressing this goal, the extension of von Stackelberg game for modelling different competing independent transmission companies would be a promising mathematical framework for a possible future with independent transmission companies.

And the last but not the least, since all concepts and theories developed throughout this paper is based on the current operation of the Eastern Australian Transmission 
system and its operating electricity market, the Author is now engaged in the application of the proposed strategy on the Eastern Australian Transmission system.

\section{APPENDIX I}

$$
\begin{aligned}
\text { Proof: } A_{L-S h a p e} & =0 \Rightarrow D_{\max }\left(\lambda_{c}+\Delta \lambda\right)-D \lambda=0 \\
& \Rightarrow \frac{\lambda_{c}+\Delta \lambda}{\lambda_{c}}=\frac{D}{D_{\max }} \Rightarrow 1+\frac{\Delta \lambda}{\lambda_{c}}=\frac{D}{D_{\max }}
\end{aligned}
$$
$\therefore \quad$ (1) $1+\frac{\Delta \lambda}{\lambda_{c}}=\frac{D}{D_{\max }}$,
(2) $\frac{D}{D_{\max }} \leq 1$,
(3) $\Delta \lambda \geq 0$

$\stackrel{(3)}{\rightarrow} \frac{\Delta \lambda}{\lambda_{c}}+1 \geq 1 \stackrel{(1)}{\rightarrow} \frac{D}{D_{\max }} \geq 1 \stackrel{(2)}{\rightarrow} D=D_{\max }$

Substituting (4) in (1) $\Rightarrow \Delta \lambda=0$

or alternatively $\left\|\lambda-\lambda_{c}\right\|_{2}=0$

\section{APPENDIX II}

The introduced vectors and matrices in (7) in terms of vectors and matrices in (6) are defined as follows;

$$
\begin{aligned}
& C=\left[\begin{array}{lll}
C^{\prime} & \text { VOLL } & 0
\end{array}\right] \\
& x^{T}=\left[\begin{array}{lll}
g & d & \theta
\end{array}\right] \\
& {[\boldsymbol{B}]=\left(\begin{array}{ccc}
{[\mathbf{0}]} & {[\mathbf{0}]} & {\left[\mathcal{H}_{l}^{\prime}\right]} \\
{[\mathbf{0}]} & {[\mathbf{0}]} & -\left[\mathcal{H}_{l}^{\prime}\right] \\
{[\boldsymbol{I}]} & {[\mathbf{0}]} & {[\mathbf{0}]} \\
-[\boldsymbol{I}] & {[\mathbf{0}]} & {[\mathbf{0}]} \\
{[\mathbf{0}]} & {[\boldsymbol{I}]} & {[\mathbf{0}]} \\
{[\mathbf{0}]} & -[\boldsymbol{I}] & {[\mathbf{0}]}
\end{array}\right)} \\
& D_{1}{ }^{T}=\left(\begin{array}{llllll}
-f_{l}^{0} & -f_{l}^{0} & -\bar{g} & \underline{g} & -\bar{d} & \underline{d}
\end{array}\right) \\
& D_{2}{ }^{T}=\left(\begin{array}{llllll}
-f_{l}^{\prime u} & -f_{l}^{\prime u} & 0 & 0 & 0 & 0
\end{array}\right) \\
& D_{3}{ }^{T}=\left(\begin{array}{llllll}
-f_{l}^{\prime e} & -f_{l}^{\prime e} & 0 & 0 & 0 & 0
\end{array}\right) \\
& \boldsymbol{A}=\left[\begin{array}{lll}
\boldsymbol{G} & \boldsymbol{D} & -\left[\mathcal{B}_{x}^{\prime}\right.
\end{array}\right]
\end{aligned}
$$

$[\boldsymbol{G}]$ and $[\boldsymbol{D}]$ are the matrices which determine the transmission connection buses of the registered generators and retailers in the electricity market.

\section{REFERENCES}

[1] "2008 ANTS consultation: final report," National Electricity Market Management Company, Sydney, NSW, Tech. Rep. Feb. 2008. [Online] Available: http://www.nemmco.com/transmission_distribution/4100099.pdf

[2] "An introduction to the Australia's national electricity market," National Electricity Market Management Company, Sydney, NSW, Tech. Rep. June 2005. [Online] available: http://nemmco.com.au/nemgeneral/0000187.pdf

[3] "Annual Planning Report 2007," Powerlink Queensland, Brisbane, QLD, Tech. Rep. Feb. 2007.[Online] Available: http://www.powerlink.com.au/data/portal/00005056/content/697100011 83076234831.pdf

[4] M.R. Hesamzadeh, N. Hosseinzadeh, P.J. Wolfs "A survey on transmission expansion planning tools in competitive electricity market" Journal of International Review of Electrical Engineering

[5] M.R. Hesamzadeh, N. Hosseinzadeh, P.J. Wolfs," Economic Assessment of Transmission Expansion Projects in Competitive Electricity Markets -An Analytical Review" Universities Power engineering Conference, Italy.

[6] P.I. Caro-ochoa, "Evaluation of the transmission congestion impact on electricity markets," M.Sc. dissertation, Dept. Elec. Eng., Univ. Illinois, 2003.

[7] R. D. Christie, B. F. Wollenberg, and I. Wangensteen, "Transmission management in deregulated environment," IEEE proceedings. No.2, vol. 88, pp. 170-195, Feb. 2000.[invited paper]

[8] A. F. Rahimi, and A. Y. Sheffrin, "Effective market monitoring in deregulated electricity market," IEEE Trans. Power Systems, vol. 18, pp. 486-493, May. 2003.

[9] M. Amelin, "On monte carlo simulation and analysis of electricity markets," Ph.D. dissertation, Dept. Elec. Eng., Royal Institute of Technology, 2004.

[10] S. Nuchprayoon, "On power scheduling and strategic behaviour in electricity markets," Ph.D. dissertation, Dept. Elec. Eng., Georgia Institute of Technology, 2003.

[11] H Von Stackelberg, The Theory of the Market Economy, Oxford University Press, Oxford 1952.

[12] B. Colson, P. Marcotte, and G. Savard, "An overview of bilevel optimization," Ann Oper Res, 153: 235-256, 2007.

[13] R. G. Jeroslow, " The polynomial hierarchy and a simple model for competitive analysis ," Mathematical Programming, 32, 146-164,1985

[14] P. Hansen, B. Jaumard, G. Savard, " New branch-and-bound rules for linear bilevel programming," SIAM Journal on Scientific and Statistical Computing, 13, 1194-1217, September 1992.

[15] J. F. Bard, Practical Bilevel Optimization, Algorithms and Applications, Netherland: Kluwer Academic Publishers, 1998.

[16] K. Shimizu, Y. Ishizuka, J. F. Bard, Nondifferentiable and Two-level Mathematical Programming, Netherland: Kluwer Academic Publishers, 1997.

[17] T. Li, M. Shahidehpour, "Strategic bidding of transmission-constrained GENCOs with incomplete information," IEEE Trans. On Power Systems. No.1, vol. 20, pp. 437-447, Feb. 2005.

[18] M. S. Bazaraa, C. M. Shetty, Linear Programming and Network Flows, vol. I. New York: Wiley, 1979

[19] M. S. Bazaraa, C. M. Shetty, Nonlinear Programming, Theory and Applications, vol. II. New York: Wiley, 1979.

[20] International Mathematical and Statistical Library, IMSL, and Intel Visual Fortran programming language

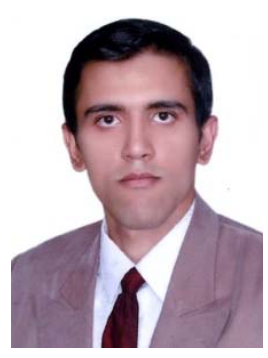

Mohammad R. Hesamzadeh (IEEE-GSM'08) is with Central Queensland University, Australia, as a Senior Researcher. His special fields of interest include high voltage transmission system design and planning, distribution and rural system studies, electricity market analysis, intelligent system applications in power systems and high voltage engineering. He is a Graduate Student Member of IEEE, Vice Chair of IEEE, Queensland Power Engineering Society, and a Professional Engineer in Australia.

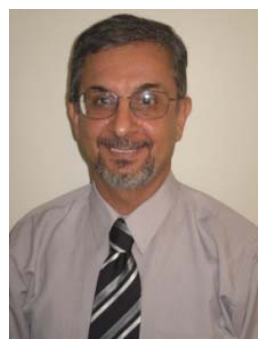

Nasser Hosseinzadeh (IEEE-M'86) is currently the Head of Department of Systems at the Faculty of Sciences, Engineering and Health at Central Queensland University, Australia. He graduated from Shiraz University, Iran, in 1986 with a B.Sc. degree in electrical and electronics engineering. $\mathrm{He}$ worked in a research centre for five years before starting his postgraduate studies. He received a M.Sc. degree from Iran University of Science and Technology in 1992 and a Ph.D. degree from Victoria University in Australia in 1998. His special 
fields of interest include power system analysis and planning, power system stability, intelligent system applications in engineering, distribution networks and rural energy supply.

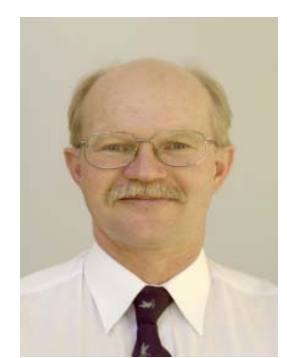

Peter J. Wolfs (IEEE-M'80-SM'99) was born in Rockhampton Australia in 1959. He graduated from the Capricornia Institute of Advanced Education in 1980 with a B.Eng. degree. He subsequently obtained the M.Eng. degree from the Philips International Institute in the Netherlands in 1981 and the Ph.D. degree at the University of Queensland in 1992. He is the Associate Dean (Research and Innovation) at the Faculty of Sciences, Engineering and Health at Central Queensland University. His special fields of interest include rural and renewable energy supply, solar and hybrid electric vehicles and intelligent systems applications in railways. Professor Wolfs is a Fellow of Engineers Australia, a Registered Professional Engineer in the State of Queensland and a member of the Railway Technical Society of Australia. 